\title{
Layer-by-Layer Deposition of Antibacterial Polyelectrolytes on Cotton Fibres
}

\author{
Ana P. Gomes $\cdot$ João F. Mano $\cdot$ João A. Queiroz $\cdot$ \\ Isabel C. Gouveia
}

Published online: 3 August 2012

(C) Springer Science+Business Media, LLC 2012

\begin{abstract}
The introduction of molecules with biological properties on textile materials is essential for a number of biotechnological applications. With the purpose of testing new processes applied to textiles, in this study, we present the first results on the feasibility of using the Layerby-Layer (LbL) deposition process in natural fibers such as cotton, with natural polyelectrolytes like chitosan $(\mathrm{CH})$ and alginic acid sodium salt (ALG), the durability of CH/ALG multilayer on cotton were evaluated. The increase of negative charges to the substrate cotton was made with $\mathrm{NaBr}$ and TEMPO, to ensure the success of the process of LbL.
\end{abstract}

\footnotetext{
A. P. Gomes

Optical Centre, University of Beira Interior,

6201-001 Covilhã, Portugal

e-mail: anapaula@ubi.pt
}

\section{J. F. Mano}

3B's Research Group, Biomaterials, Biodegradables and

Biomimetics, Headquarters of the European Institute of

Excellence on Tissue Engineering and Regenerative Medicine,

University of Minho, AvePark, 4806-909 Taipas, Guimarães,

Portugal

e-mail: jmano@dep.uminho.pt

\section{J. F. Mano}

ICVS/3B's, PT Government Associate Laboratory,

Braga, Guimarães, Portugal

\section{J. A. Queiroz}

Health Sciences Research Centre, University of Beira Interior, 6201-001 Covilhã, Portugal

e-mail: jqueiroz@ubi.pt

\section{C. Gouveia ( $\square)$}

R\&D Unit of Textile and Paper Materials, Faculty of

Engineering, University of Beira Interior,

6201-001 Covilhã, Portugal

e-mail: igouveia@ubi.pt
Three characterization methods to assess electrostatic LbL deposition were performed: the contact angle between a liquid (water) and the sample surface, in order to characterize the wettability of the samples with the different layers of $\mathrm{CH}$ and ALG; dyeing of the $\mathrm{CH} / \mathrm{ALG}$ assembled cotton fabric with cationic methylene blue that shows regular changes in terms of color depth ( $K / S$ value), which indicate that the surface were alternately deposited with $\mathrm{CH}$ and ALG layers and, finally, the analysis by infrared spectroscopy using Fourier Transform with Attenuated Total Reflection (ATR-FTIR), to assess the changes in the interaction between $\mathrm{CH}$ and ALG deposited on cotton samples.

Keywords Layer-by-layer - Contact angle .

ATR-FTIR · Chitosan · Alginate

\section{Introduction}

The challenges facing the textile finishing industry have intensified during the last decade. Current awareness of the negative environmental impact of chemical processing of textiles, combined with increased strict legislation on industrial effluents, has led to the search for advanced, nonpolluting processes for coating textiles. Coating on textiles is a new way to give functionalities and properties on textile surfaces without compromising on fabric properties and they open a whole new vista of value-addition possibilities in the textile sector. The coating can be used to give wrinkle resistance, improve color or light fastness, flame retardancy, water or oil repellency and antibacterial properties [1]. Newer methods of coating textiles become possible to improve the functionality and durability of the coating to a higher level compared to the conventional 
coating techniques. These techniques include immobilization of enzymes, LbL assemblies, nano coatings and use of plasma for deposition of functional molecules. All these techniques are distinct from conventional finishes in that they impart special functionalities to textile surfaces by bringing about modifications at micro or nano level, without affecting the bulk properties. These processes add functionality with minimum effect on the strength, feel, handle or breathability of textiles [2]. Some of these techniques have been tested and validated at lab scale, but most are still in research stages. Most of the conventional coating techniques either affect the fabric flexibility, comfort and permeability or deteriorate the mechanical properties of the treated fabric [3, 4].

Functional coating methods provide a flexible alternative to conventional finishing methods in that they are independent of fabric type, require low quantities of additives and allow combinations of different functionalities in a simple way [2]. Polyelectrolyte multilayer (PEM) coatings have become a new and general way to functionalize surfaces [5]. This technique has been described as being theoretically able to cover many kinds of surfaces when they are charged [6]. The mechanisms allowing for film coating essentially involve electrostatic interactions, but the assembly of such multilayer structures have also been show on non-ionic or apolar substrates [7]. The film is constructed by the alternate adsorption of oppositely charged polyelectrolytes at the surface of the material, easily obtained when the material is dipped in polyelectrolyte solutions. The driving force for the film construction is related with the excess charge (alternatively positive and negative) which appears after each new polyelectrolyte adsorption $[8,9]$. A deposition cycle creates a bilayer, and these cycles can be repeated as often as needed. The number of deposition cycles and the types of polyelectrolytes used in the construction allow for the control of thickness and roughness of the multilayered film [10]. A broad range of applications for these films has been considered, going from drug delivery to specific bio-applications based on surface modifications. For example, the multilayer film technique has been used to create microcapsules, defined as micro and nano-containers for storage, transport, and release of active macromolecules [11]. Martins et al. analyzed the potential and achievements of LbL technique as a promising approach to functionalize biomaterials surface in a controllable and facile manner. They found that the build-up of CH/ALG system presented a linear growth, meaning that no polymer has the ability to diffuse "in" and "out" of the film after each deposition step [9].

A new method for the modification of textile fibers was introduced by Hyde et al. [12], they demonstrated that a polymer thin film could be deposited directly onto cotton fabrics by following the widely studied LbL deposition method known as PEM. The LbL method involves essentially electrostatic interactions. Using transmission electron microscopy (TEM) they have shown the uniform coating provided by the LbL onto the cotton fabric [12]. It seems possible that by following the same LbL deposition method, a wide range of molecules, nanoparticles, and other functionalized polyelectrolyte could be deposited on textile fabric leading towards the development of new applications for technical textile applications. So the LbL self assembly method may provide new coatings or films that can be constructed by the alternate adsorption of oppositely charged polyelectrolytes at the surface of the material, easily obtained when the material is dipped in polyelectrolyte solutions. LbL is a simple and inexpensive method for preparation of controlled layered structures and it is applicable to a variety of materials. It has the advantages of simplicity, low cost, ability to incorporate different bio molecules and molecular control. LbL film structures are less densely packed and this is advantageous for diffusion through the films [13]. But certain details of the process are still not clearly understood. The LbL deposition process has not been extensively implemented in textile and natural fibers, as they possess unique challenges including the chemical heterogeneity of their surfaces as well as their irregular shapes.

Numerous studies involving different polymer substrates and several synthetic polyelectrolytes have been published. But, there are very few scientific articles concerning the deposition of alternate polyelectrolyte on natural textile supports. During a deep revision, we found few reports concerning the LbL method involving cotton fibres.

Hyde et al. [14], they evaluated three different levels of cotton cationization. Variations in the cationization degree were achieved by manipulating the ratio of 3-cloro-2hydroxy propyl trimethyl ammonium to $\mathrm{NaOH}$. The deposition of the polyelectrolytes was monitored using XPS and CHNS elemental analysis. The experimental results they obtained, indicated that the deposition process was not significantly influenced by the degree of cotton cationization. The build-up of further polyelectrolyte layers was found to be less sensitive to variations in the cationic character of the substrate once a critical number of alternating layers was deposited [14].

Wang et al. [15], they have utilized two different methods for the characterization for LbL deposition of two polyelectrolytes poly (sodium styrene sulfonate) (PSS) and poly (dimethyldiammonium chloride) (PDDA) on cotton fabrics, a dyeing method and a UV absorption method. Two types of dyes, anionic Direct Red 80 and cationic Methylene Blue, were utilized to dye the self-assembled cotton in order to reveal the change of surface electric property after LbL deposition of polyelectrolytes on cotton. 
The UV absorption method could monitor the growth of polyelectrolytes on cotton substrates in terms of UV absorbance at characteristic absorption wavelengths [15].

Wang et al. [16], they have studied a new approach for UV protection of cotton fabrics based on LbL self-assembly. Three fluorescent brightening agents and polycation PDDA were used on cationized cotton fabrics through direct LbL deposition technique. The assembled cotton fabrics could obtain excellent rating of UV protection when the fluorescent brightening agents and PDDA were built up on the cotton substrates. Good durability to washing revealed the stability of multilayers films on the cationized cotton, which is important for actual application of textiles [16].

Ugur et al. [17], they obtained $\mathrm{ZnO}$ nanoparticle-based multilayer nanocomposite films were fabricated on cationized woven cotton fabrics via LbL deposition process. In this study they concluded that the process by LbL provides a novel and simple method for nano- $\mathrm{ZnO}$ nanocomposite film deposition on cotton fabrics and their application onto cotton fabrics to gain antibacterial and UV protection functions [17].

Ali et al. [3] developed a study to evaluate the effect of different process parameters on the amount of polyelectrolyte adsorbed on a cotton textile substrate via sequential adsorption of negatively charged PSS and positively charged poly allylamine hydrochloride (PAH) using LbL [3].

Recently Ali et al. [18] deposited CH nanocoating onto a cotton textile substrate using a LbL technique. PSS, was used as the anionic polyelectrolyte, and $\mathrm{CH}$ was used as the cationic polyelectrolyte. As a result, they obtained a uniform surface deposition of bilayers, as observed by scanning electron microscopy (SEM) and confirmed that during the LbL deposition, the layers did not block the fabric pores (unlike conventional coatings) [18].

As the commonly used polyelectrolytes such as PSS, poly (acrylic acid) (PAA), PDDA, poly(allylamine hydrochloride) (PAH) and polyethyleneimine (PEI) have no special functions transferred to textiles [12, 15, 16, 19-22] non-polyelectrolytes with negative charges such as nanoparticles and dyestuffs were integrated into the LbL selfassembled multilayers together with polyelectrolytes to modify the surface giving antibacterial properties and dyeability, respectively [16]. It should be noted that this new technique is not limited to polyelectrolytes. Some organic molecules with positive or negative charges have also been integrated into multilayers via LbL deposition. This opens the possibility of developing functional textiles. Therefore, here we report the first results regarding the feasibility of LbL deposition of nanolayers of natural bioactive polyelectrolytes, to give antibacterial properties to natural fibers. The purpose of this experimental work is to determine the feasibility of using the LbL deposition process in natural fibers with natural polyelectrolytes. To impart higher negative charge to the substrate cotton, $\mathrm{CH}$ and ALG were successfully layered over cotton fibers using LbL technique.

The introduction of negative charges onto cotton samples is made with sodium bromide, $\mathrm{NaBr}$ and 2,2,6,6-tetramethylpiperidinyl-1-oxy free radical (TEMPO) [23-26]. The cotton is composed for the most part of cellulose, cellulose is a natural polymer composed of $\beta$-D-glucopyranose units that are linked together by $(1 \rightarrow 4)$ - glycosidic bonds [23]. A cellulose molecular chain, depending on the source of the cellulose, consists of 300-15,000 D-glucose units. The unit has three hydroxyl groups on $\mathrm{C} 2, \mathrm{C} 3$ and C6, respectively, and the hydroxyl group of C6 is much more reactive than that of $\mathrm{C} 2$ and $\mathrm{C} 3$. TEMPO is a stable nitroxide radical, which can catalytically oxidize primary and secondary alcohols under aqueous condition with high selectivity and efficiency. In this study we follow the oxidation method that uses a mixture of sodium hypochlorite, sodium bromide and TEMPO. With such reagents, the oxidation is selective as it oxidizes exclusively the primary hydroxyl groups while leaving untouched the secondary ones [23, 24, 26, 27]. There are several works where TEMPO-mediated oxidation was applied to cellulose fibers under various conditions with good results [23, 27-29].

The number of biofunctional textiles with antibacterial activity has increased considerably over the last few years [30-32]. Application is now extended to biomedical products, which is perhaps the largest application of antibacterial textiles $[33,34]$. There is a wide range of methods available to examine the interaction of microorganisms with textiles. In order to evaluate the activity of antibacterial textiles there are several standard methods available. The most common can be divided into two categories: (1) qualitative methods: AATCC 147:1998 and JIS L 1902:2002-Halo method and (2) quantitative methods: AATCC 100:1999 and JIS L 1902:2002-Absorption method. In the qualitative method, textile samples are placed onto agar plates, which have been inoculated with bacteria and are then incubated under moist conditions at $37^{\circ} \mathrm{C}$ for $24-48 \mathrm{~h}$. The intention is that intimate contact between the textile, the bacteria, and the growth medium will result in the inhibition of growth either immediately adjacent to the textile or in an area around the textile. A new approach to evaluate the effectiveness of the antibacterial activity of textile fibers as well as bacteria adhesion on textiles by using SEM is described in a previous paper [35]. The SEM analysis revealed great potential on the evaluation and effectiveness of antibacterial activity of textiles. Also, the bacterial adhesion and the morphology of bacteria after exposure to antibacterial agents, was determined using the same. 


\section{Experimental}

Layer by Layer Coating of Cotton

Cotton fabrics obtained from James H. Heal \& Co. Ltd was used as substrate, cotton samples were used with dimensions of $2 \mathrm{~cm} \times 2 \mathrm{~cm}$. TEMPO, $\mathrm{NaBr}$, Sodium Hypochlorite $(\mathrm{NaOCl}) 5 \%, \mathrm{CH}$ (low molecular weight), Acetic Acid $\left(\mathrm{CH}_{3} \mathrm{COOH}\right), \mathrm{ALG}$, Sodium Chloride $(\mathrm{NaCl})$, Sodium Hydroxide $(\mathrm{NaOH})$ and Hydrochloric acid $(\mathrm{HCl})$ were purchased from Sigma-Aldrich. All chemicals were of analytical grade and used as received.

Antibacterial polyelectrolyte $\mathrm{CH}(1 \mathrm{mg} / \mathrm{mL})$ and ALG $(1 \mathrm{mg} / \mathrm{mL})$ solutions were prepared by dissolving $\mathrm{CH}$ and ALG in $0.1 \mathrm{M} \mathrm{CH}_{3} \mathrm{COOH}$ and $0.5 \mathrm{M} \mathrm{NaCl}$ solutions, respectively. The $\mathrm{pH}$ values were adjusted to 5 using $0.1 \mathrm{M}$ $\mathrm{HCl}$ and $1 \mathrm{~N} \mathrm{NaOH}$ solutions. The $\mathrm{pH}$ was selected to 5, to be approximately intermediate between the $\mathrm{pK}_{\mathrm{a}}$ of $\mathrm{CH}$ (6.3) and ALG, $\mathrm{pK}_{\mathrm{a}}$ of 3.38 and 3.65 [36]. ALG and $\mathrm{CH}$ are two oppositely charged natural polyelectrolyte materials and very sensitive toward changes in external factor such as $\mathrm{pH}$. At $\mathrm{pH} 5$ the carboxylate group of ALG mainly exists in the form of $\mathrm{COO}^{-}$and the amino group of $\mathrm{CH}$ mainly exists in the form of $\mathrm{NH}_{3}{ }^{+}$. In this case the presence of both $\mathrm{COO}^{-}$and $\mathrm{NH}_{3}{ }^{+}$along polymer backbone could enhance the electrostatic interaction of the network structure [37]. This value of $\mathrm{pH} 5$ was used in several works in the process of LbL with $\mathrm{CH}$ and ALG [38-43].

To apply LbL technique, two samples of the substrate (cotton) were charged by immersing cotton samples in $(\mathrm{TEMPO}+\mathrm{NaBr}+\mathrm{NaClO} 5 \%, \mathrm{pH}=10.5)$ solution under moderate stirring for 30 and $120 \mathrm{~min}$, respectively, followed by a rinse with deionized water, as described elsewhere [26, 28]. Then, $\mathrm{CH}$ and ALG polyelectrolytes multilayer films were deposited over cotton by the LbL assembly whereas $\mathrm{CH}$ was used as polycation and ALG as polyanion. For each layer deposition, the cotton substrate was immersed into the corresponding solution at room temperature and for $5 \mathrm{~min}$, followed by rinsing with deionized water to remove excessive polyelectrolyte. Since cotton samples were charged negatively, the $\mathrm{CH}$ was deposited as the first layer. Samples were prepared with five layers (CH/ALG/CH/ALG/CH), six layers (CH/ALG/ $\mathrm{CH} / \mathrm{ALG} / \mathrm{CH} / \mathrm{ALG}$ ), nine layers (CH/ALG/CH/ALG/CH/ $\mathrm{ALG} / \mathrm{CH} / \mathrm{ALG} / \mathrm{CH})$ and ten layers $(\mathrm{CH} / \mathrm{ALG} / \mathrm{CH} / \mathrm{ALG} /$ $\mathrm{CH} / \mathrm{ALG} / \mathrm{CH} / \mathrm{ALG} / \mathrm{CH} / \mathrm{ALG})$, respectively in control samples functionalized by LbL (designated by B5, B6, B9 and B10), cotton samples treated during $30 \mathrm{~min}$ in TEMPO and then functionalized by LbL (designated by CT5, CT6, CT9 and CT10) and finally cotton samples treated during $120 \mathrm{~min}$ in TEMPO and then functionalized by LbL (designated by 2CT5, 2CT6, 2CT9 and 2CT10). After the last deposition, the sample was dried in a desiccator at room temperature overnight. Control samples were also prepared using the same method by LbL, without pretreatment with TEMPO.

The functionalized samples were washed and tested the durability of the CH/ALG multilayers. The durability to washing of samples functionalized by LbL was determined following the NP 1710, textiles-wash fastness test (Portuguese Standard). The Fig. 1 show the SEM images of the cotton (control), the $\mathrm{CH} / \mathrm{ALG} / \mathrm{CH} / \mathrm{ALG} / \mathrm{CH} / \mathrm{ALG}$ (six layers) sample and the sample that were washed after $\mathrm{LbL}$ deposition. The functionalized samples show a large and heterogeneous deposition of polyelectrolytes, Fig. 1b. In contrast, the washed samples show a less but more uniform polyelectrolytes deposition Fig. 1c.

The results indicate that the cotton assembled with $\mathrm{CH} /$ ALG multilayers had good durability to washing. Good durability to washing revealed the stability of multilayer films on the cotton surface, which is important in various applications of textiles, is not important for application in disposable materials.

\section{Control Tests}

\section{Contact Angle}

The measurement of the contact angle between water and sample surface is one of the easiest ways to characterize the
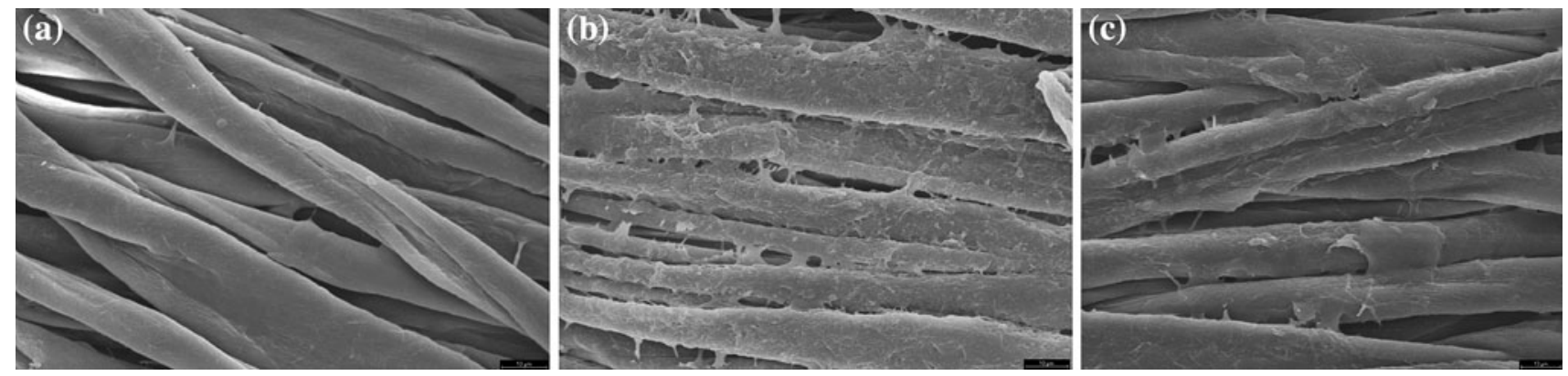

Fig. 1 SEM images. (a) Cotton sample, (b) CT6 and (c) CT6 after washed 
wettability of the material [44]. A hydrophilic or hydrophobic surface is defined by these contact angle values. For values higher than $90^{\circ}$ the surface is hydrophobic and less than $90^{\circ}$ it is hydrophilic [45].

The sessile drop contact angle method [46] was used to measure the contact angles of a water drop on the functionalized cotton samples with a contact angle system Dataphysics model OCAH 200. Contact angles were determined by placing $5 \mu \mathrm{l}$ drops of deionized water. The tip of the needle was removed, and then an image was recorded. Average values were obtained from multiple contact angle values (at least six) on each sample using an optical system connected to a video display. The measures presented were made at $100,1,000$ and $10,000 \mathrm{~ms}$, according to standard TAPPI T 558 pm-95 "Surface wettability and absorbency of sheeted materials using an automated contact angle tester".

\section{Control Method for Evaluation of Negatively Charged Layers}

The present study attempts to evaluate the effect of different process parameters on the amount of polyelectrolyte adsorbed on a cotton samples via sequential adsorption of positively charged $\mathrm{CH}$ and negatively charged ALG using LbL self-assembly process. The amount of polyelectrolyte adsorption on cotton fabric was evaluated by measuring the color value $(K / S)$ of methylene blue absorbed cotton surface $[15,16,19,20,47]$. Methylene blue exhibit adsorption is proportional to the amount of anionic groups on fibers [48]. In cotton there is abundance of carbohydrate hydroxyl groups, methods of characterization of these groups are in constant development. Several analytical techniques are being applied and new ones introduced and tested, in this paper we use the adsorption of methylene blue. This dye was extensively studied as an indicator of the amount of anionic groups on fibers $[15,16,19,20,47$, 48] A dyeing method in terms of $K / S$ values has been proven a simple and quick means to characterize the change of surface polarities of LbL self-assembled textile substrates based on the attraction or repulsion between the cationic dye and polyelectrolytes. The relative color depth of the dyed fabrics expressed as $K / S$, was measured by the light reflectance technique using the Kubelka-Munk equation [15].

$K / S=\frac{(1-R)^{2}}{2 R}$

Kubelka-Munk theory describes optical characteristics (e.g. reflectance, transmittance and absorbance) by a variety of light scattering media including paints, textiles and paper $[49,50]$. The reflectance $(R)$ of the dyed fabrics was measured at the maximum absorbance wavelength on a
Datacolor Spectraflash SF300. The ratio of the absorption coefficient $(K)$ to the scattering coefficient $(S)$ varies with the total light reflected according to above expression. To determine $K$, it is necessary to first compute $\mathrm{S}$, which involves measurements either of the transmittance of the thickness, or of the reflectance of the thickness against backgrounds of known reflectance. If it could be assumed that $S$ was reasonably constant under a given set of experimental conditions, then $K / S$ values should be proportional to the amount of dye and could be used in much the same way that absorbance values were used in the analysis of samples by transmitted light, higher dye content leads to an increase in absorbance. Therefore, the $K / S$ values were expected to be proportional to the amount of dye [51] The values obtained of $K / S$ are commonly used to represent the amount of dye fixation or dye content of a given textile fibers [47].

Cotton specimens with different numbers of layers were dyed using $7.5 \%$ owf cationic dye (methylene blue) [16]. The dyeing was performed in petri dishes without stirring at temperature of $40^{\circ} \mathrm{C}$ for $15 \mathrm{~min}$. After immersion in the dye solution, the samples were soaked in deionized water and air dried.

\section{ATR-FTIR Analysis}

The study was made using Fourier Transform Infrared Spectroscopy in Attenuated Total Reflection mode (ATRFTIR) with a Vertex 70 spectrophotometer. The transmittance was converted into absorbance for display.

ATR-FTIR reveals information about the molecular structure of chemical compounds and is useful for the characterization of biopolymers. The carbonyl vibrations of a carboxylate and a carboxylic acid group occur at different wave numbers, as does the $\mathrm{N}-\mathrm{H}$ vibrations of amines and protonated amines [43]. These analyses were made in order to investigate the success of the LbL technique applied to samples of cotton, through the existence or absence of functional groups with specific vibrations.

\section{Results and Discussion}

\section{Contact Angle}

To identify and distinguish the wettability of each layer in the $\mathrm{CH} / \mathrm{ALG}$ multilayer film, samples were prepared as described in Table 1. Since the last assembled layer has the most significant effect on the surface property, according to the outermost layer is CH or ALG [52]. The surface wettability is very sensitive to the surface compositions of the outermost layer. The sessile drop contact angle obtained on various surfaces was shown in Table 1. This table presents 
Table 1 Contact angle

\begin{tabular}{|c|c|c|c|c|}
\hline Sample $n^{\circ}$ & Description of sample & $100 \mathrm{~ms}$ & $1,000 \mathrm{~ms}$ & $10,000 \mathrm{~ms}$ \\
\hline 1 & Cotton & $47.52 \pm 11.72$ & - & - \\
\hline 2 (B5) & Control sample-with $\mathrm{CH} / \mathrm{ALG} / \mathrm{CH} / \mathrm{ALG} / \mathrm{CH}$ & $86.13 \pm 13.93$ & $55.56 \pm 18.33$ & - \\
\hline 3 (B6) & Control sample-with $\mathrm{CH} / \mathrm{ALG} / \mathrm{CH} / \mathrm{ALG} / \mathrm{CH} / \mathrm{ALG}$ & - & - & - \\
\hline 4 (B9) & Control sample-with $\mathrm{CH} / \mathrm{ALG} / \mathrm{CH} / \mathrm{ALG} / \mathrm{CH} / \mathrm{ALG} / \mathrm{CH} / \mathrm{ALG} / \mathrm{CH}$ & $42.85 \pm 10.14$ & - & - \\
\hline $5(\mathrm{~B} 10)$ & Control sample-with $\mathrm{CH} / \mathrm{ALG} / \mathrm{CH} / \mathrm{ALG} / \mathrm{CH} / \mathrm{ALG} / \mathrm{CH} / \mathrm{ALG} / \mathrm{CH} / \mathrm{ALG}$ & - & - & - \\
\hline $6(\mathrm{CT})$ & Anionic cotton (30 min TEMPO), CT & $23.31 \pm 4.89$ & - & - \\
\hline 7 (CT5) & $\mathrm{CT}$-with $\mathrm{CH} / \mathrm{ALG} / \mathrm{CH} / \mathrm{ALG} / \mathrm{CH}$ & $114.6 \pm 4.46$ & $106.17 \pm 12.93$ & - \\
\hline 8 (CT6) & $\mathrm{CT}$-with $\mathrm{CH} / \mathrm{ALG} / \mathrm{CH} / \mathrm{ALG} / \mathrm{CH} / \mathrm{ALG}$ & $67.67 \pm 13.10$ & - & - \\
\hline 9 (СТ9) & $\mathrm{CT}$-with $\mathrm{CH} / \mathrm{ALG} / \mathrm{CH} / \mathrm{ALG} / \mathrm{CH} / \mathrm{ALG} / \mathrm{CH} / \mathrm{ALG} / \mathrm{CH}$ & $114.63 \pm 10.13$ & $101.53 \pm 17.27$ & - \\
\hline $10(\mathrm{CT} 10)$ & $\mathrm{CT}$-with $\mathrm{CH} / \mathrm{ALG} / \mathrm{CH} / \mathrm{ALG} / \mathrm{CH} / \mathrm{ALG} / \mathrm{CH} / \mathrm{ALG} / \mathrm{CH} / \mathrm{ALG}$ & $82.65 \pm 12.07$ & $38.6 \pm 2.97$ & - \\
\hline $11(2 \mathrm{CT})$ & Anionic cotton (120 min TEMPO), 2CT & $22.25 \pm 14.50$ & - & - \\
\hline $12(2 \mathrm{CT} 5)$ & 2CT- with $\mathrm{CH} / \mathrm{ALG} / \mathrm{CH} / \mathrm{ALG} / \mathrm{CH}$ & $122.58 \pm 7.76$ & $123.08 \pm 3.99$ & $116.95 \pm 10.53$ \\
\hline 13 (2CT6) & 2CT- with $\mathrm{CH} / \mathrm{ALG} / \mathrm{CH} / \mathrm{ALG} / \mathrm{CH} / \mathrm{ALG}$ & $54.98 \pm 6.20$ & $17.35 \pm 4.17$ & - \\
\hline 14 (2CT9) & 2CT- with $\mathrm{CH} / \mathrm{ALG} / \mathrm{CH} / \mathrm{ALG} / \mathrm{CH} / \mathrm{ALG} / \mathrm{CH} / \mathrm{ALG} / \mathrm{CH}$ & $112.57 \pm 9.50$ & $115.62 \pm 6.97$ & $113.23 \pm 7.55$ \\
\hline 15 (2CT10) & 2CT—with $\mathrm{CH} / \mathrm{ALG} / \mathrm{CH} / \mathrm{ALG} / \mathrm{CH} / \mathrm{ALG} / \mathrm{CH} / \mathrm{ALG} / \mathrm{CH} / \mathrm{ALG}$ & $58.4 \pm 8.17$ & - & - \\
\hline
\end{tabular}

the contact angle of cotton (sample $n^{\circ} 1$ ), control samples functionalized by LbL (samples $\mathrm{n}^{\circ} 2-5$, designated by: B5, B6, B9, B10), anionic cotton treated during $30 \mathrm{~min}$ in TEMPO (sample $\mathrm{n}^{\circ} 6, \mathrm{CT}$ ), previous anionic cotton functionalized by LbL (samples $\mathrm{n}^{\circ} 7-10$, designated by: CT5, CT6, CT9, CT10), anionic cotton treated during $120 \mathrm{~min}$ in TEMPO (sample $\mathrm{n}^{\circ} 11,2 \mathrm{CT}$ ) and previous anionic cotton functionalized by LbL (samples $\mathrm{n}^{\circ} 12-15$, designated by: 2CT5, 2CT6, 2CT9, 2CT10). Results show that for the control samples (samples B5, B6, B9, B10) untreated with TEMPO, the contact angle values are much lower than those of the other samples. Cotton fibers contain abundant hydroxyl groups, making the fiber surface highly hydrophilic. Although cotton fibers are known to be charged slightly negatively because of the ionization of some hydroxyl groups [53], the TEMPO-oxidation method is selective as it oxidizes exclusively the primary hydroxyl group [24], so the TEMPO-oxidation makes the cotton fibers charge enough for the electrostatic assembly. For cotton, the initial contact angle was around $47.52^{\circ}$, for sample CT and 2CT the contact angle suffered a decrease because of the hydrophilic character of cotton when immersed in TEMPO, due to the formation of a greater number of negative charges on the sample surface. In general, the water contact angle decreases as the extent of surface charges increases and the surface becomes more hydrophilic. For all samples functionalized by LbL, the greatest reduction in contact angle was observed for the samples CT6, CT10, 2CT6 and 2CT10, confirming the presence of permanent negative charges in the polymer chains (ALG) as a generator of a hydrophilic matrix. For the samples CT5, CT9, 2CT5 and 2CT9, where the last layer is $\mathrm{CH}$, higher contact angles were achieved, showing the hydrophobic character of $\mathrm{CH}$. This is in accordance with previous studies, where the $\mathrm{CH}$ layer deposition led to a contact angle increase $[52,54,55]$. The different values of contact angle according to the outermost layer is $\mathrm{CH}$ or ALG which are similar to those found in literature, pure $\mathrm{CH}$ membranes were more hydrophobic than ALG membranes [54-60].

Figure 2 shows the contact angle (measured at $100 \mathrm{~ms}$ ) of the multilayer films with layer number, from 0 (cotton sample), 5, 6, 9 and 10, for samples treated $30 \mathrm{~min}$ and $120 \mathrm{~min}$ in TEMPO. For all the conditions, the contact angle exhibits the zigzag feature with the layer number, indicating the alternate assembly of $\mathrm{CH}$ and ALG on the surface. These observations are due to the surface

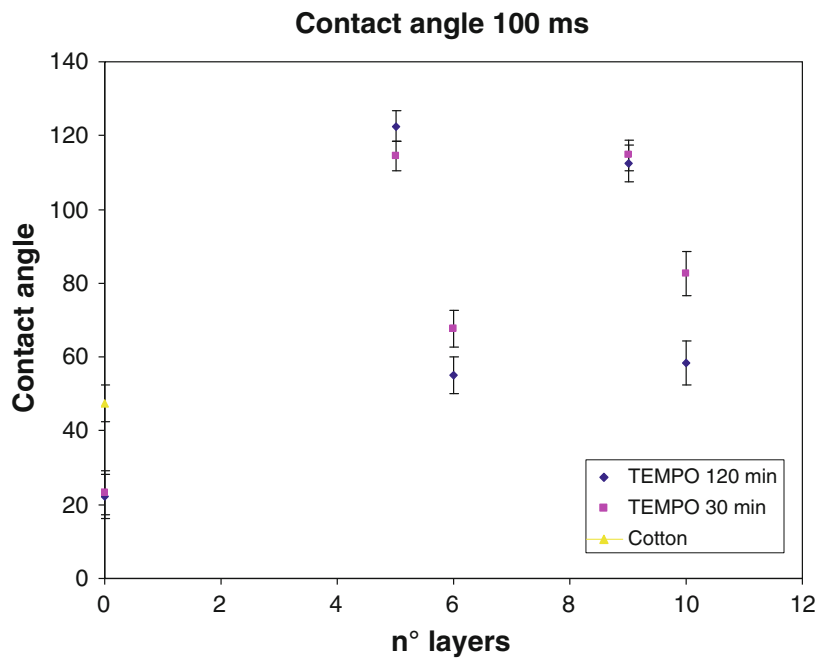

Fig. 2 Contact angle of CH/ALG multilayer films with 5, 6, 9 and 10 numbers of layers. The sample without $\mathrm{LbL}$ functionalized is layer zero 
composition as a result of the contact angle of $\mathrm{CH}$ that is bigger than that of the ALG. Typically, the ALG layer induced an decrease of contact angle whereas $\mathrm{CH}$ layer deposition led to a contact angle increase. The wettability of a surface depends on the nature of the outermost layer and not on the initial substrate film [61].

\section{Control Method for Evaluation of Negatively Charged Layers}

The electrical properties of the cotton surface would change alternately between positively charged and negatively charged after LbL electrostatic assembly of polyelectrolytes. So, it is valuable to determine the change in surface polarity of assembled cotton to demonstrate the stepwise buildup of CH/ALG multilayers. The cationic methylene blue was used to dye the self-assembled cotton in order to reveal the change of cotton surface. The increase in absorbance at $600 \mathrm{~nm}$ of the samples was monitored using a reflectance spectrophotometer. We can use the increase of the $K / S$ value as a characteristic of the LbL deposition of the dye on fibers. The growth of multilayers on the cotton surface based on LbL self-assembly was assessed in terms of the UV change absorbance of assembled substrates.

As shown in Fig. 3 the regular changes in color depth of assembled cotton samples indicate that the surfaces were alternately deposited with every $\mathrm{CH}$ and ALG layers. As the outermost layer alternated between $\mathrm{CH}$ and ALG, the $K / S$ values present a regular oscillations, revealing that the surface is covered by $\mathrm{CH}$ or by ALG which is enough to change the surface polarities. The high $K / S$ values shown in Fig. 3 indicate a high level of adhesion of the ALG on the

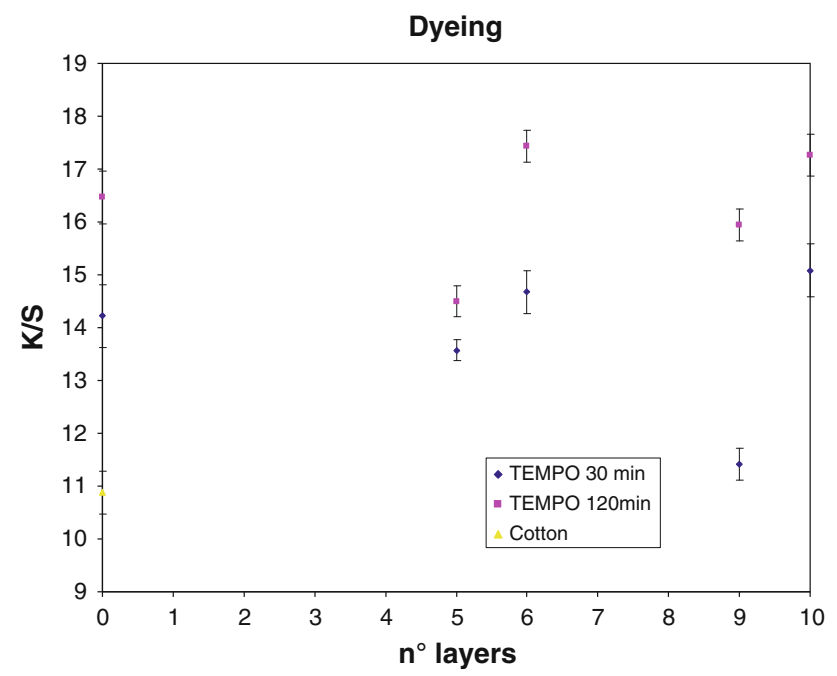

Fig. 3 Tracing of the color depth $(K / S)$ versus the number of monolayers in CH/ALG assembled multilayers on cotton dyed with methylene blue. The first layer is $\mathrm{CH}$ and the surface layer alternates between $\mathrm{CH}$ and ALG sample. Moreover, the fact that the data measured at four different locations, including both sides of the fabric, was nearly the same demonstrates that the surface is uniformly coated. When $\mathrm{CH}$ first layer is deposited on activated cotton, a part of the $\mathrm{NH}^{+}$groups in $\mathrm{CH}$ were bound with anionic groups of cotton through ionic bonds, the remained would make the cotton surface present net positive charges. The newly formed positively charged surface rejects the absorption of cationic direct dye because of the repulsion between the same charges, resulting in a decrease of color depth. However, when ALG was subsequently assembled on the $\mathrm{CH}$ coated cotton surface, the electric properties of cotton surface were reversed. The newly formed cotton surface with net negative charges would attract the cationic dye, causing the increase of $K / S$ values. The composition of the polyelectrolytes deposited on the surface of assembled cotton directly relates to the linear increases in UV absorbance. Therefore, the growth of these multilayers could be recorded by monitoring the UV spectra of assembled cotton specimens. The behavior observed in Fig. 3, where the $K / S$ value exhibits a zigzag feature through even/odd layer numbers, indicating the alternate assembly of $\mathrm{CH}$ and ALG on the surface, is consistent with other results found in the literature $[15,16]$.

The electrical properties of the cotton surface changed alternately between positively charged and negatively charged after LbL electrostatic assembly of polyelectrolytes. Therefore, the change in surface polarity of assembled cotton demonstrates the stepwise fabrication of $\mathrm{CH} / \mathrm{ALG}$ multilayers. As the outermost layer alternated between $\mathrm{CH}$ and ALG the $K / S$ values presented regular oscillations, revealing that the surface coverage of $\mathrm{CH}$ by ALG and vice versa is enough to change the surface polarities.

We initially used the method for evaluation of negatively charged in samples of cotton and cotton pre-treated with TEMPO. In Fig. 4b we found that the sample has a more intense color than in Fig. 4a. This is due to the cotton sample pre-treated with TEMPO get negative charges, so there is a greater absorption to the surface of the cationic dye methylene blue. The complexity of textile surface makes the monitoring of the multilayers become difficult, because the textile substrates in fabric form have a nonplanar surface. Considering the change of surface electric property of the cotton specimen during self-assembly procedure, which had been demonstrated by determining the color depth of cotton surface, it can be concluded that $\mathrm{CH}$ were produced on the cotton substrate via bonding with oppositely charged ALG.

\section{ATR-FTIR}

In the current study, an LbL assembly was produced on a cotton fabric to explore the interaction between $\mathrm{CH}$ and 
Fig. 4 Images for evaluation of negatively charged layers. a Cotton sample with methylene blue; b cotton sample pretreated with TEMPO
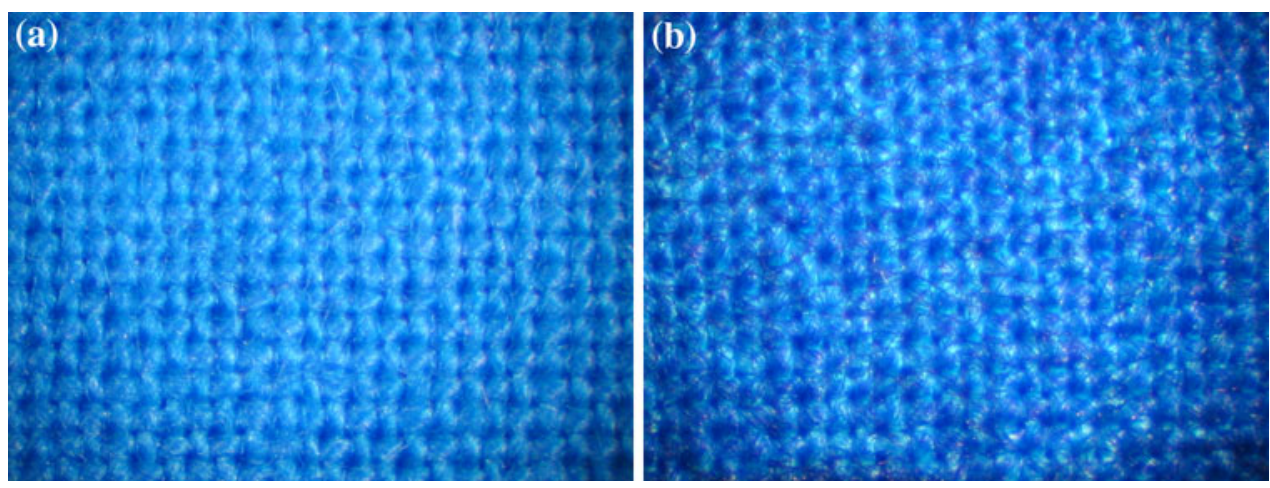

ALG. In order to evaluate the ability of the functionalization process with TEMPO to produce a surface capable of supporting polyelectrolyte films via LbL deposition, ATRFTIR spectrum of the received cotton, Fig. 5a, functionalized cotton samples when immersed $30 \mathrm{~min}$ in TEMPO (CT sample), Fig. 5b and functionalized cotton samples when immersed $120 \mathrm{~min}$ in TEMPO (2CT sample), Fig. 5c, were assessed. When cotton was immersed into TEMPO, Fig. 5b, c and took new spectrum of the functionalized cotton, the spectrum was almost identical to the original. Figure 5, shows characteristic cellulose peaks around 1,000-1,200 $\mathrm{cm}^{-1}$, which are the main components of cotton [62-64]. As shown in Fig. 5, in the current study, the absorbance intensity of the characteristic peak at around $1,600 \mathrm{~cm}^{-1}$ varied. The band is absent in the cotton sample (Fig. 5a), but can be detected in CT and 2CT samples (Fig. 5b, c). A maximum, but not significant value for this band is reached in the spectrum (Fig. 5c) for the 2CT sample, corresponding to the maximum oxidation conditions. Thus, we can conclude that the TEMPOmediated oxidation conditions were selectively converted to carboxylate ion ionized form $\left(\mathrm{COO}^{-}\right)$, imparting a negative surface charge to the cotton, as discussed previously [62]. The TEMPO-mediated oxidation treatment was

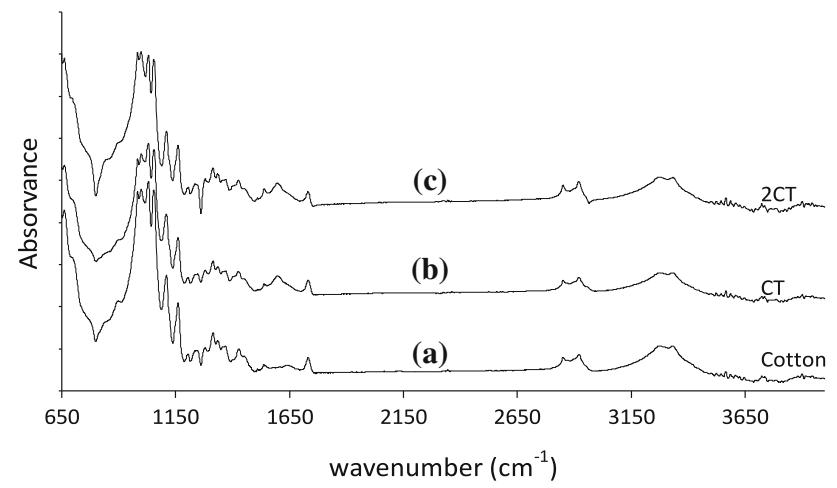

Fig. 5 ATR-FTIR spectra of cotton and of cotton TEMPO- oxidized with different immersion times. (a) Cotton sample, $(b)$ immersed $30 \mathrm{~min}$ in TEMPO, $(c)$ immersed $120 \mathrm{~min}$ in TEMPO proven to be able of modifying a surface of samples cotton to produce a surface capable of supporting multilayer films.

Figure 6 shows the ATR-FTIR spectra of cotton and cotton assembled with $\mathrm{CH} / \mathrm{ALG}$ multilayers. In this figure six regions can be distinguished: first at $770-830 \mathrm{~cm}^{-1}$, $1,180-1,300 \mathrm{~cm}^{-1}, 1,330-1,450 \mathrm{~cm}^{-1}, 1,600-1,800 \mathrm{~cm}^{-1}$, $2,800-2,980 \mathrm{~cm}^{-1}$ and finally at $3,100-3,550 \mathrm{~cm}^{-1}$. Figure 6 showed characteristic cellulose peaks around $1,000-1,200 \mathrm{~cm}^{-1}$ [64]. Other characteristic bands related to the chemical structure of cellulose were hydrogenbonded $\mathrm{OH}$ stretching around $3,100-3,550 \mathrm{~cm}^{-1}$, the $\mathrm{C}-\mathrm{H}$ stretching around $2,800 \mathrm{~cm}^{-1}$ and the asymmetrical $\mathrm{COO}^{-}$ stretching around $1,600 \mathrm{~cm}^{-1}[53,62-64]$. If the carboxylate existed in ionized form $\left(\mathrm{COO}^{-}\right)$, it would show two peaks at 1,600 and $1,400 \mathrm{~cm}^{-1}$ for the asymmetric and the symmetric stretching of $\mathrm{COO}^{-}$ion, respectively [62]. In carboxylate ion, if it is protonated, it would become $-\mathrm{COOH}$ in which double bond $(\mathrm{C}=\mathrm{O})$ and single bond $(\mathrm{C}-\mathrm{OH})$ would exist. The $\mathrm{C}=\mathrm{O}$ stretching would show at around $1,750 \mathrm{~cm}^{-1}$ and $\mathrm{C}-\mathrm{OH}$ stretching at $1,200 \mathrm{~cm}^{-1}$ $[62,64]$. This was confirmed by the spectrum of samples shown in Fig. 6. Based on several studies [41-43, 65] the characteristic peaks of $\mathrm{CH}$ were detected in a region around $1,700-1,500 \mathrm{~cm}^{-1}$ corresponding to amino group. The

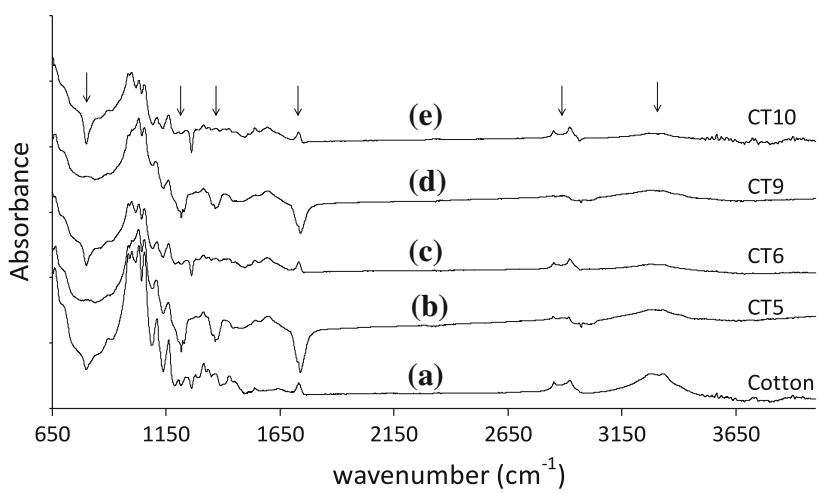

Fig. 6 ATR-FTIR spectra of cotton (a) and samples CT5 (b), CT6 $(c)$, CT9 $(d)$ and CT10 (e). Arrows indicates the six regions can be distinguished 
ALG spectrum shows characteristic band of carboxylate $\left(\mathrm{COO}^{-}\right)$band at 1,600 and $1,400 \mathrm{~cm}^{-1}$. The observation of theses peaks in Fig. 6, indicated that the degree of ionic interaction between the negatively charged carboxylic ion group of ALG and the positively charged amino group of $\mathrm{CH}$. The study of Alves et al. and Lawrie et al. [41, 43] present values of the characteristic stretching bands in ATR-FTIR spectrum which correspond to $\mathrm{CH}$ and ALG related to the chemical structure. When $\mathrm{CH}$ is the outermost layer in an LbL assembly (Fig. 6b, d), CT5 and CT9 samples, the amine groups extending into solution during fabrication will become deprotonated upon washing and drying (around 1,400 $\mathrm{cm}^{-1}$ ).

However, when ALG is the outermost layer (Fig. 6c, e), CT6 and CT10 samples, the amine groups of the underlying $\mathrm{CH}$ layer will be protonated to a larger degree due to interaction with the deprotonated carboxylate groups of ALG. It was therefore expected that a higher amount of protonated amines will be present when ALG is the outmost layer $[41,43]$. This behavior is observed in the peak around 1,400 and 1,200 $\mathrm{cm}^{-1}$ in Fig. 6. It can be seen in Fig. 6 that after the assembly of 5 bilayers, the absorption band around $3,300 \mathrm{~cm}^{-1}$, corresponding to the hydroxyl groups of cellulose, became less evident, this result also confirms the presence of $\mathrm{CH} / \mathrm{ALG}$ multilayers on cotton. The same study was done for samples 2CT5, 2CT6, 2CT9 and 2CT10, and it was found that the analysis of spectra was identical to that described previously. The fourth region in the FTIR spectrum at $1,680-1,800 \mathrm{~cm}^{-1}$, Fig. $7 \mathrm{~b}, \mathrm{~d}$ the spectrum for samples B5 and B9 have the same behavior that samples CT5, 2CT5, CT9 and 2CT9, but for samples B6 and B10 the behavior is the inverse of the samples CT6, 2CT6, CT10 and 2CT10. On the other hand, for this last group of samples, on the second region $\left(1,180-1,300 \mathrm{~cm}^{-1}\right)$ and third region $\left(1,330-1,450 \mathrm{~cm}^{-1}\right)$ the band disappeared. The behavior of ATR-FTIR spectrum of control samples is exactly the same in the various layers, this does not happen in samples CT5, CT6, CT9 and CT10, comparatively to spectra of Fig. 6. This indicates that the LbL process was

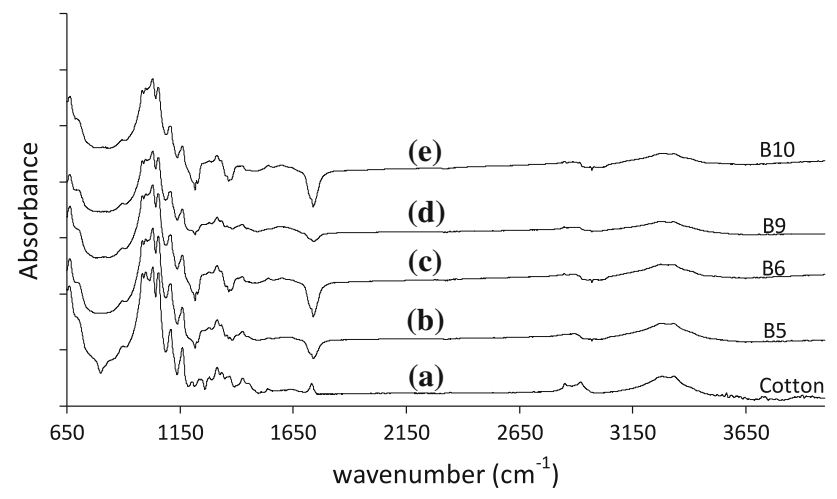

Fig. 7 ATR-FTIR spectra of cotton (a) and control samples $(b-e)$ not successful in these samples, because these samples were not activated with TEMPO-oxidation. Two different levels of TEMPO-oxidation (30 and $120 \mathrm{~min}$ ) were used to functionalize cotton samples substrate in order to investigate the role of the supporting surface in the buildup of the multilayer. According to the results, we found that the activation of the substrate with TEMPO-oxidation is necessary to ensure the success of the LbL technique applied to cotton. Concerning the level of oxidation, there are no relevant differences in results for of the immersion times of 30 and $120 \mathrm{~min}$ in TEMPO-oxidation. Be noted that some characteristic peaks of absorption could not almost be identified from assembled cotton, even after a (CH/ALG) multilayer was fabricated on cotton. This might be due to the fact that ATR-FTIR is not sensitive enough to the small amount of the deposited materials. In addition, partial overlapping of characteristic absorption peaks between the functionalized cotton and the polyelectrolytes might also result in the above phenomenon [15].

The samples used in this study have been tested on the antibacterial properties on a previous study [35]. Antibacterial activity of functionalized cotton samples was determined in terms of inhibition zone formed around the sample and analyzed by SEM. Control samples showed low antibacterial activity and bacterial growth on the surface of control samples analyzed by SEM indicated the presence of colonies. LbL functionalized cotton sample presented a clear area around it with no bacterial growth (zone of inhibition). Analyzing these samples by SEM, we observed damaged bacteria under and around them.

\section{Conclusions}

The LbL deposition process was used to deposit alternate layers of $\mathrm{CH}$ and ALG on cotton substrates. Treatment of the cotton samples with TEMPO was proven to be an effective procedure to create a substrate able to support multilayer films. This result was confirmed by various methods of analysis used in this work. The activation of the substrate with TEMPO-oxidation is necessary to ensure the success of the LbL technique applied to cotton.

The surface wettability is very sensitive to the surface compositions of the outermost layer. Using the contact angle method it was found that differently functionalized samples presented different values. Samples where the last layer was $\mathrm{CH}$ had a higher value of contact angle compared to samples having ALG on the last layer. This result is consistent with the considerations found in the literature, classifying $\mathrm{CH}$ has having hydrophobic character and ALG has having hydrophilic character. This fact demonstrated the formation of alternating layers of $\mathrm{CH}$ and $\mathrm{ALG}$, indicating that the process of LbL was successfully applied in cotton samples. 
Dyeing of the CH/ALG assembled cotton samples with cationic methylene blue shows regular and observable "odd-even" changes in terms of color depth $(K / S$ value), indicating the variation of surface composition of the cotton substrates due to the alternate deposition of $\mathrm{CH}$ and ALG on them.

ATR-FTIR provided direct and indirect evidence of the efficacy of the deposition process. It was possible to follow the formation of the multilayers of $\mathrm{CH} / \mathrm{ALG}$ on cotton samples, analyzing the chemical changes in each layer. These experimental results validate the feasibility of using the LbL self-assembled deposition of natural polyelectrolytes on cotton substrates as a novel processing method for textile functionalization.

The LbL of CH/ALG on cotton textile has potent antibacterial activity toward both Gram-positive and Gramnegative bacteria. These coating cotton textiles are potentially useful as antibacterial fabrics in a wide variety of biomedical and general use applications.

Overall, the results showed a promising eco-friendly and simple technique to give functionalized textiles with antibacterial properties using natural polyelectrolytes with antibacterial agents. This method can open new avenues towards the development of non-toxic and safe biomedical textiles.

Excellent durability to washing of the CH/ALG multilayers was obtained, which indicates good adhesion between the multilayer coatings and the cotton surfaces.

Acknowledgments The authors wish to acknowledge the Fundação para a Ciência e Tecnologia and Compete for the financial support of the project: PTDC/EBB-BIO/113671/2009 Skin2Tex.

\section{References}

1. Joshi M, Ali SW, Agarwal N (2009) Functional nanocoatings on textiles through layer-by-layer self assembly. In: Proceedings of the fiber society 2009 spring conference, vols I and Ii, pp 956

2. Gulrajani ML, Gupta D (2011) Emerging techniques for functional finishing of textiles. Indian J Fibre Text Res 36(4):388-397

3. Ali SW, Rajendran S, Joshi M (2010) Effect of process parameters on layer-by-layer self-assembly of polyelectrolytes on cotton substrate. Polym Polym Comp 18(5):175-187

4. Gowri $\mathrm{S}$ et al (2010) Polymer nanocomposites for multifunctional finishing of textiles-a review. Text Res J 80(13):1290-1306

5. Richert L et al (2004) Layer by layer buildup of polysaccharide films: physical chemistry and cellular adhesion aspects. Langmuir 20(2):448-458

6. Decher G (1997) Fuzzy nanoassemblies: toward layered polymeric multicomposites. Science 277(5330):1232-1237

7. Chen W, McCarthy TJ (1997) Layer-by-layer deposition: a tool for polymer surface modification. Macromolecules 30(1):78-86

8. Decher G, Hong JD, Schmitt J (1992) Buildup of ultrathin multilayer films by a self-assembly process. 3. Consecutively alternating adsorption of anionic and cationic polyelectrolytes on charged surfaces. Thin Solid Films 210(1-2):831-835
9. Martins GV et al (2010) Crosslink effect and albumin adsorption onto chitosan/alginate multilayered systems: an in situ QCM-D study. Macromol Biosci 10(12):1444-1455

10. Picart C et al (2001) Determination of structural parameters characterizing thin films by optical methods: a comparison between scanning angle reflectometry and optical waveguide lightmode spectroscopy. J Chem Phys 115(2):1086-1094

11. Ibarz G et al (2001) Smart micro- and nanocontainers for storage, transport, and release. Adv Mater 13(17):1324-1327

12. Hyde K, Rusa M, Hinestroza J (2005) Layer-by-layer deposition of polyelectrolyte nanlayers on natural fibres: cotton. Nanotechnology 16(7):S422-S428

13. Ariga K, Hill JP, Ji QM (2008) Biomaterials and Biofunctionality in Layered Macromolecular Assemblies. Macromol Biosci 8(11):981-990

14. Hyde K, Dong H, Hinestroza JP (2007) Effect of surface cationization on the conformal deposition of polyelectrolytes over cotton fibers. Cellulose 14(6):615-623

15. Wang Q, Hauser PJ (2009) New characterization of layer-bylayer self-assembly deposition of polyelectrolytes on cotton fabric. Cellulose 16(6):1123-1131

16. Wang Q, Hauser PJ (2010) Developing a novel UV protection process for cotton based on layer-by-layer self-assembly. Carbohydr Polym 81(2):491-496

17. Ugur SS et al (2010) Modifying of cotton fabric surface with nano-ZnO multilayer films by layer-by-layer deposition method. Nanoscale Res Lett 5(7):1204-1210

18. Ali SW, Joshi M, Rajendran S (2011) Novel, self-assembled antimicrobial textile coating containing chitosan nanoparticles. Aatcc Rev 11(5):49-55

19. Polowinski S (2005) Polyelectrolyte layer-by-layer processed coated textiles. Fibres Text Eastern Eur 13(6):50-52

20. Jantas R, Polowinski S (2007) Modifying of polyester fabric surface with polyelectrolyte nanolayers using the layer-by-layer deposition technique. Fibres Text Eastern Eur 15(2):97-99

21. Polowinski S (2007) Deposition of polymer complex layers onto nonwoven textiles. J Appl Polym Sci 103(3):1700-1705

22. Stawski D, Bellmann C (2009) Electrokinetic properties of polypropylene textile fabrics containing deposited layers of polyelectrolytes. Colloids Surf Physicochem Eng Aspects 345(1-3):191-194

23. Saito $\mathrm{T}$ et al (2005) Distribution of carboxylate groups introduced into cotton linters by the TEMPO-mediated oxidation. Carbohydr Polym 61(4):414-419

24. Montanari S et al (2005) Topochemistry of carboxylated cellulose nanocrystals resulting from TEMPO-mediated oxidation. Macromolecules 38(5):1665-1671

25. Isogai A et al (2006) TEMPO-mediated oxidation of native cellulose: microscopic analysis of fibrous fractions in the oxidized products. Carbohydr Polym 65(4):435-440

26. Dang Z, Zhang JG, Ragauskas AJ (2007) Characterizing TEMPO-mediated oxidation of ECF bleached softwood kraft pulps. Carbohydr Polym 70(3):310-317

27. Praskalo J et al (2009) Sorption properties of TEMPO-oxidized natural and man-made cellulose fibers. Carbohydr Polym 77(4):791-798

28. Saito $\mathrm{T}$ et al (2006) TEMPO-mediated oxidation of native cellulose: microscopic analysis of fibrous fractions in the oxidized products. Carbohydr Polym 65(4):435-440

29. Saito T, Isogai A (2004) TEMPO-mediated oxidation of native cellulose. The effect of oxidation conditions on chemical and crystal structures of the water-insoluble fractions. Biomacromolecules 5(5):1983-1989

30. Singh R et al (2005) Antimicrobial activity of some natural dyes. Dyes Pigm 66(2):99-102 
31. Gao Y, Cranston R (2008) Recent advances in antimicrobial treatments of textiles. Text Res J 78(1):60-72

32. Papaspyrides CD, Pavlidou S, Vouyiouka SN (2009) Development of advanced textile materials: natural fibre composites, antimicrobial, and flame-retardant fabrics. In: Proceedings of the institution of mechanical engineers part L-journal of materialsdesign and applications, vol 223(L2), p 91-U2

33. Kramer A et al (2006) Hygienic relevance and risk assessment of antimicrobial-impregnated textiles. Curr Probl Dermatol 33: 78-109

34. Zilberman M, Elsner JJ (2008) Antibiotic-eluting medical devices for various applications. J Controlled Release 130(3):202-215

35. Gomes A, Mano J, Queiroz J, Gouveia I (2010) Assessement of bacteria-textile interactions using scanning electron microscopy: a study on LbL chitosan/alginate coated cotton. In Méndez-Vilas A (ed) Microscopy: science, technology, applications and education. Formatex, Spain, pp 286-292

36. Maurstad G et al (2008) Polyelectrolyte layer interpenetration and swelling of alginate-chitosan multilayers studied by dual wavelength reflection interference contrast microscopy. Carbohydr Polym 71(4):672-681

37. Ding Y et al (2009) Assembled alginate/chitosan micro-shells for removal of organic pollutants. Polymer 50(13):2841-2846

38. Xie YL, Wang MJ, Yao SJ (2009) Preparation and characterization of biocompatible microcapsules of sodium cellulose sulfate/chitosan by means of layer-by-layer self-assembly. Langmuir 25(16):8999-9005

39. Zhao QH et al (2006) Assembly of multilayer microcapsules on $\mathrm{CaCO} 3$ particles from biocompatible polysaccharides. J Biomaterials Sci Polym Edition 17(9):997-1014

40. Yang Y et al (2007) Assembled alginate/chitosan nanotubes for biological application. Biomaterials 28(20):3083-3090

41. Alves NM, Picart C, Mano JF (2009) Self assembling and crosslinking of polyelectrolyte multilayer films of chitosan and alginate studied by QCM and IR spectroscopy. Macromol Biosci 9(8):776-785

42. Deng HB et al (2010) Layer-by-layer structured polysaccharides film-coated cellulose nanofibrous mats for cell culture. Carbohydr Polym 80(2):474-479

43. Lawrie $\mathrm{G}$ et al (2007) Interactions between alginate and chitosan biopolymers characterized using FTIR and XPS. Biomacromolecules 8(8):2533-2541

44. Khalil-Abad MS, Yazdanshenas ME (2010) Superhydrophobic antibacterial cotton textiles. J Colloid Interface Sci 351(1): 293-298

45. Rotta J et al (2009) Parameters of color, transparency, water solubility, wettability and surface free energy of chitosan/ hydroxypropylmethylcellulose (HPMC) films plasticized with sorbitol. Mater Sci Eng C-Biomimetic Supramol Syst 29(2):619623

46. Kwok DY, Neumann AW (1999) Contact angle measurement and contact angle interpretation. Adv Colloid Interface Sci 81(3): 167-249

47. Dubas ST, Kumlangdudsana P, Potiyaraj P (2006) Layer-by-layer deposition of antimicrobial silver nanoparticles on textile fibers. Colloids Surf Physicochem Eng Aspects 289(1-3):105-109
48. Peterlin S et al (2009) Dyeing of papermaking fibers with dyes of various structural types as a means for fiber surface characterization. Acta Chim Slov 56(2):418-425

49. Kokhanovsky AA (2007) Physical interpretation and accuracy of the Kubelka-Munk theory. J Phys D Appl Phys 40(7):2210-2216

50. Kubelka P (1948) New contributions to the optics of intensely light-scattering materials. J Optical Soc Am 38(5):448-457

51. ElSherif M, Bayoumi OA, Sokkar TZN (1997) Prediction of absorbance from reflectance for an absorbing-scattering fabric. Color Res Appl 22(1):32-39

52. Yuan WY et al (2007) pH-controlled construction of chitosan/ alginate multilayer film: characterization and application for antibody immobilization. Langmuir 23(26):13046-13052

53. Zhao Y et al (2010) Superhydrophobic cotton fabric fabricated by electrostatic assembly of silica nanoparticles and its remarkable buoyancy. Appl Surf Sci 256(22):6736-6742

54. Hsu SH et al (2004) Chitosan as scaffold materials: Effects of molecular weight and degree of deacetylation. J Polym Res Taiwan 11(2): 141-147

55. Xie HG et al (2010) Effect of surface wettability and charge on protein adsorption onto implantable alginate-chitosan-alginate microcapsule surfaces. J Biomed Mater Res Part A 92A(4): $1357-1365$

56. Liu YL et al (2007) Chitosan/poly(tetrafluoroethylene) composite membranes using in pervaporation dehydration processes. J Membr Sci 287(2):230-236

57. Rane AB et al (2009) Formulation and evaluation of press coated tablets for pulsatile drug delivery using hydrophilic and hydrophobic polymers. Chem Pharm Bull 57(11):1213-1217

58. Oddo L et al (2010) Novel thermosensitive calcium alginate microspheres: physico-chemical characterization and delivery properties. Acta Biomater 6(9):3657-3664

59. Mengatto L, Luna JA, Cabrera MI (2010) Influence of crosslinking density on swelling and estradiol permeation of chitosan membranes. J Mater Sci 45(4):1046-1051

60. Yao BL et al (2010) Hydrophobic modification of sodium alginate and its application in drug controlled release. Bioprocess Biosyst Eng 33(4):457-463

61. Carneiro-Da-Cunha MG et al (2010) Physical and thermal properties of a chitosan/alginate nanolayered PET film. Carbohydr Polym 82(1):153-159

62. Chung C, Lee M, Choe EK (2004) Characterization of cotton fabric scouring by FT-IR ATR spectroscopy. Carbohydr Polym 58(4):417-420

63. Wang Q et al (2006) Characterization of bioscoured cotton fabrics using FT-IR ATR spectroscopy and microscopy techniques. Carbohydr Res 341(12):2170-2175

64. Yan HJ et al (2009) Analysis of the chemical composition of cotton seed coat by Fourier-transform infrared (FT-IR) microspectroscopy. Cellulose 16(6):1099-1107

65. Dai $\mathrm{M}$ et al (2009) Chitosan-alginate sponge: preparation and application in curcumin delivery for dermal wound healing in rat. J Biomed Biotechnol 2009:1-8 